\title{
Non-homogenous $\mathrm{KdV}$ and coupled sub-ballistic fractional PDEs
}

\author{
Arman Aghili \\ Department of applied Mathematics, Faculty of Mathematical sciences, University of Guilan, Rasht, Iran \\ Received: 7 November 2016, Accepted: 10 March 2017 \\ Published online: 25 August 2017.
}

\begin{abstract}
In this article, the author solved certain system of time fractional equations using integral transforms. Transform method is a powerful tool for solving singular integral equations, evaluation of certain integrals and solution to partial fractional differential equations. The result reveals that the transform method is very convenient and effective.
\end{abstract}

Keywords: Caputo fractional derivative, nonhomogeneous time fractional heat equation, Laplace transform, Fourier transform, linear KdV equation.

\section{Introduction}

In recent years, it has turned out that many phenomena in fluid mechanics, physics, biology, engineering and other areas of sciences can be successfully modeled by the use of fractional derivatives. Fractional differential equations arise in unification of diffusion and wave propagation phenomenon. The time fractional heat equation, which is a mathematical model of a wide range of important physical phenomena, is a partial differential equation obtained from the classical heat equation by replacing the first time derivative by a fractional derivative of order In this work, we considered methods and results for the system of partial fractional differential equations which arise in applications.

Several methods have been introduced to solve fractional differential equations, the popular Laplace transform method, [1],[2], [3],[12], the Fourier transform method [11], the iteration method [18] and operational method [11],[6]. However, most of these methods are suitable for special types of fractional differential equations, mainly the linear with constant coefficients. More detailed information about some of these results can be found in a survey paper by Kilbas and Trujillo[10] Atanackovic and Stankovic [4],[5] and Stankovic [20] used the Laplace transform in a certain space of distributions to solve a system of partial differential equations with fractional derivatives, and indicated that such a system may serve as a certain model far a visco elastic rod.

Oldham and Spanier I [13] and [14], respectively, by reducing a boundary value problem involving Fickâ's second law in electro-analytic chemistry to a formulation based on the partial Riemann Liouville fractional with half derivative. Oldham and Spanier [14] gave other application of such equations for diffusion problems. Schneider [19] and Wyss [22] considered the time fractional diffusion and wave equations and obtained the solution in terms of Fox functions. 


\section{Definitions and notations}

Definition 1. The left Caputo fractional derivative of order $\alpha$ $(0<\alpha<1)$ of $\phi(t)$ is as follows[8]

$$
D_{a}^{c, \alpha} \phi(x)=\frac{1}{\Gamma(1-\alpha)} \int_{a}^{t} \frac{1}{(t-\xi)^{\alpha}} \phi^{\prime}(\xi) d \xi
$$

Definition 2. The Laplace transform of function $f(t)$ is defined as follows

$$
\mathscr{L}\{f(t)\}=\int_{0}^{\infty} e^{-s t} f(t) d t=F(s)
$$

If $\mathscr{L}\{f(t)\}=F(s)$, then $\mathscr{L}^{-1}\{F(s)\}$ is given by

$$
f(t)=\frac{1}{2 \pi i} \int_{c-i \infty}^{c+i \infty} e^{s t} F(s) d s
$$

where $F(s)$ is analytic in the region $\operatorname{R} e(s)>c$. The above integral is known as Bromwich complex inversion formula.

Example 1. By using an appropriate integral representation for the modified Bessel's functions of the second kind of order $v, K_{v}(s)$, show that

$$
\mathscr{L}^{-1}\left\{\frac{K_{\eta}(a \sqrt{s})}{s^{\frac{\eta}{2}}} \frac{K_{v}(b \sqrt{s})}{s^{\frac{v}{2}}}\right\}=\frac{t^{\eta-1} e^{-\frac{a^{2}}{4 t}}}{(2 a)^{1+\eta}} * \frac{t^{v-1} e^{-\frac{b^{2}}{4 t}}}{(2 b)^{1+v}} .
$$

Solution 1. It is well known that $K_{v}(a \sqrt{s})$ has the following integral representation [6]

$$
K_{v}(a \sqrt{s})=\frac{(a \sqrt{s})^{v}}{2^{v+1}} \int_{0}^{\infty} e^{-\xi-\frac{a^{2} s}{4 \xi}} \frac{d \xi}{\xi^{v+1}}
$$

at this point, using complex inversion formula for the Laplace transform and the above integral representation we get

$$
\mathscr{L}^{-1}\left\{\frac{K_{\eta}(a \sqrt{s})}{s^{\frac{\eta}{2}}}\right\}=\frac{1}{2 i \pi} \int_{c-i \infty}^{c+i \infty} \frac{e^{t s}}{s^{\eta}}\left(\frac{(\sqrt{a s})^{2 \eta}}{2^{\eta+1}} \int_{0}^{\infty} e^{-\xi-\frac{a^{2} s}{4 \xi}} \frac{d \xi}{\xi \eta+1}\right) d s .
$$

Changing the order of integration and simplifying to get

$$
\mathscr{L}^{-1}\left\{\frac{K_{\eta}(a \sqrt{s})}{s^{\frac{\eta}{2}}}\right\}=a^{\eta} \int_{0}^{\infty} \frac{e^{-\xi}}{\xi \eta+1}\left(\frac{1}{2 i \pi} \int_{c-i \infty}^{c+i \infty} \frac{e^{\left(t-\frac{a^{2}}{4 \xi}\right) s}}{2^{\eta+1}} d s\right) d \xi .
$$

The value of inner integral is $\delta\left(t-\frac{a^{2}}{4 \xi}\right)$, we arrive at

$$
\mathscr{L}^{-1}\left\{\frac{K_{\eta}(a \sqrt{s})}{s^{\frac{\eta}{2}}}\right\}=a^{\eta} \int_{0}^{\infty} \frac{e^{-\xi}}{\xi^{\eta+1}} \delta\left(t-\frac{a^{2}}{4 \xi}\right) d \xi .
$$

making a change of variable $\left(t-\frac{a^{2}}{4 \xi}\right)=u$, and using elementary properties of Dirac - delta function, we get the following result

$$
\mathscr{L}^{-1}\left\{\frac{K_{\eta}(a \sqrt{s})}{s^{\frac{\eta}{2}}}\right\}=\frac{t^{\eta-1} e^{-\frac{a^{2}}{4 t}}}{(2 a)^{\eta+1}} .
$$


Finally, we obtain

$$
\mathscr{L}^{-1}\left\{\frac{K_{\eta}(a \sqrt{s})}{s^{\frac{\eta}{2}}} \frac{K_{V}(b \sqrt{s})}{s^{\frac{v}{2}}}\right\}=\frac{t^{\eta-1} e^{-\frac{a^{2}}{4 t}}}{(2 a)^{\eta+1}} * \frac{t^{\nu-1} e^{-\frac{b^{2}}{4 t}}}{(2 b)^{v+1}} .
$$

Definition 3. The The Laplace transform of Caputo fractional derivatives of order non integer . For $n-1<\alpha \leq n$, we have the following identity [15][16]

$$
\mathscr{L}\left\{{ }_{0}^{C} D_{t}^{\alpha} f(t)\right\}=s^{\alpha} F(s)-\sum_{k=0}^{n-1} s^{\alpha-k-1} f^{(k)}(0) .
$$

Definition 4. The The two-parameter function of the Mittag-Leffler type is defined by the series expansion

$$
E_{\alpha, \beta}(z)=\sum_{n=0}^{\infty} \frac{z^{n}}{\Gamma(\alpha n+\beta)} .
$$

when $\alpha, \beta, z \in C$. We have the following relationship

$$
\mathscr{L}\left\{t^{\beta-1} E_{\alpha, \beta}\left( \pm a t^{\alpha}\right)\right\}=\frac{s^{\alpha-\beta}}{s^{\alpha} \mp a}\left(\operatorname{Re}(s)>|a|^{\frac{1}{\alpha}} .\right.
$$

Definition 5. The simplest Wright function is given by the series

$$
W(\alpha, \beta ; z)=\sum_{n=0}^{\infty} \frac{z^{n}}{n ! \Gamma(\alpha n+\beta)},
$$

when $\alpha, \beta, z \in C$. We have the following relationship

$$
\mathscr{L}\left\{t^{\beta-1} E_{\alpha, \beta}\left( \pm a t^{\alpha}\right)\right\}=\frac{s^{\alpha-\beta}}{s^{\alpha} \mp a}\left(R e(s)>|a|^{\frac{1}{\alpha}}\right) .
$$

Theorem 1. (Schouten-Van der Pol Theorem) Consider a function $f(t)$ which has the Laplace transform $F(s)$ which is analytic in the half-plane $\operatorname{Re}(s)>s_{0}$. We can use this knowledge to find $g(t)$ whose Laplace transform $G(s)$ equals $F(\phi(s))$, where $\phi(s)$ is also analytic for $\operatorname{Re}(s)>s_{0}$. This means that if

$$
G(s)=F(\phi(s))=\int_{0}^{\infty} f(\tau) \exp (-\phi(s) \tau) d \tau,
$$

and

$$
g(t)=\frac{1}{2 \pi i} \int_{c-i \infty}^{c+i \infty} F(\phi(s)) \exp (t s) d s,
$$

then

$$
g(t)=\int_{0}^{\infty} f(\tau)\left(\frac{1}{2 \pi i} \int_{c-i \infty}^{c+i \infty} \exp (-\phi(s) \tau) \exp (t s) d s\right) d \tau
$$

Proof. See [6]. 
Lemma 1. The following identity holds true

$$
g(t)=\mathscr{L}^{-1}\{F(\sqrt{s})\}=\int_{0}^{\infty} \frac{\tau}{2 \sqrt{\pi t^{3}}} e^{-\frac{\tau^{2}}{4 t}} f(\tau) d \tau .
$$

Proof. If we set $\phi(s)=\sqrt{s}$ in relation (16), it leads to

$$
G(s)=F(\sqrt{s})=\int_{0}^{\infty} f(\tau) \exp (-\sqrt{s} \tau) d \tau
$$

and

$$
g(t)=\frac{1}{2 i \pi} \int_{c-i \infty}^{c+i \infty} F(\sqrt{s}) \exp (t s) d s
$$

then, we get

$$
g(t)=\int_{0}^{\infty} f(\tau)\left(\frac{1}{2 \pi i} \int_{c-i \infty}^{c+i \infty} \exp (-\sqrt{s} \tau) \exp (t s) d s\right) d \tau
$$

after evaluation of the inner integral by means of table of the Laplace transforms, we arrive at

$$
g(t)=\mathscr{L}^{-1}\{F(\sqrt{s})\}=\int_{0}^{\infty} \frac{\tau}{2 \sqrt{\pi t^{3}}} e^{-\frac{\tau^{2}}{4 t}} f(\tau) d \tau .
$$

Lemma 2. Let us assume that $\mathscr{L}\{f(t)\}=F(s)$, then we have the following relations

(1). $\mathscr{L}\left\{f\left(t^{2}\right)\right\}=\int_{0}^{\infty} \sqrt{\frac{1}{4 \pi \xi}} e^{-\frac{s^{2}}{4 \xi}} F(\xi) d \xi$,

(2). $\mathscr{L}\left\{f\left(\frac{1}{t}\right)\right\}=\int_{0}^{\infty} \sqrt{\frac{\xi}{s}} J_{1}(2 \sqrt{s \xi}) F(\xi) d \xi$,

(3). $\mathscr{L}\left\{f\left(t^{3}\right)\right\}=\int_{0}^{\infty} \sqrt{\frac{s}{\xi}} K_{\frac{1}{3}}\left(\left(\frac{s}{3 \sqrt[3]{\xi}}\right)^{\frac{2}{3}}\right) F(\xi) d \xi$.

Proof. See [6].

Note.The above Lemma has some interesting applications as below.

Lemma 3. The following integral relations hold true.
(1). $\int_{0}^{\infty} K_{\frac{1}{3}}\left(\left(\frac{1}{3 \sqrt[3]{\xi}}\right)^{\frac{2}{3}}\right) \frac{d \xi}{\xi \sqrt{\xi} \sqrt[3]{\xi^{2}}}=\frac{9 \pi}{2 \Gamma\left(\frac{2}{3}\right)}$
(2). $\int_{0}^{\infty} \sqrt{\frac{\xi}{s}} J_{1}(2 \sqrt{s \xi}) e^{-\lambda \xi} d \xi=\frac{e^{-s \lambda}}{\lambda^{2}}$.

Proof. (1). Let us take $f(t)=t^{\frac{2}{3}}$, then we get $F(s)=\frac{\Gamma\left(\frac{5}{3}\right)}{s^{\frac{5}{3}}}$, on the other hand we have

$$
\left.\mathscr{L}\left\{f\left(t^{3}\right)\right\}=\mathscr{L}\left\{t^{2}\right)\right\}=\frac{2}{s^{3}}
$$

By setting all of the information in part 3 of the Lemma 1, we get the following

$$
\mathscr{L}\left\{f\left(t^{3}\right)\right\}=\frac{1}{3 \pi} \int_{0}^{\infty} \sqrt{\frac{s}{\xi}} K_{\frac{1}{3}}\left(\left(\frac{s}{3 \sqrt[3]{\xi}}\right)^{\frac{2}{3}}\right) \frac{\Gamma\left(\frac{5}{3}\right)}{\xi^{\frac{5}{3}}} d \xi=\frac{2}{s^{3}}
$$


Now, by choosing $s=1$ and after some simple manipulations we arrive at

$$
\int_{0}^{\infty} \sqrt{\frac{1}{\xi}} K_{\frac{1}{3}}\left(\left(\frac{1}{3 \sqrt[3]{\xi}}\right)^{\frac{2}{3}}\right) \frac{1}{\xi^{\frac{5}{3}}} d \xi=\frac{9 \pi}{2 \Gamma\left(\frac{2}{3}\right)}
$$

(2). Let us take $f(t)=\delta(t-\lambda)$, then we have $F(s)=e^{-\lambda s}$. On the other hand $f\left(\frac{1}{t}\right)=\delta\left(\frac{1}{t}-\lambda\right)$, and the Laplace transform of $\delta\left(\frac{1}{t}-\lambda\right)$ is as follows

$$
\mathscr{L}\left\{f\left(\frac{1}{t}\right)\right\}=\int_{0}^{\infty} \delta\left(\frac{1}{t}-\lambda\right) e^{-s t} d t
$$

In order to evaluate the above integral, we introduce a change of variable $w=\frac{1}{t}-\lambda$, to get

$$
\mathscr{L}\left\{f\left(\frac{1}{t}\right)\right\}=\int_{-\lambda}^{\infty} \delta(w) e^{-\frac{s}{w+\lambda}} \frac{d w}{(w+\lambda)^{2}}=\frac{e^{-\frac{s}{\lambda}}}{\lambda^{2}}
$$

using second part of the Lemma1.2. we obtain

$$
\mathscr{L}\left\{f\left(\frac{1}{t}\right)\right\}=\int_{0}^{\infty} \sqrt{\frac{\xi}{s}} J_{1}(2 \sqrt{s \xi}) F(\xi) d \xi=\frac{e^{-\frac{s}{\lambda}}}{\lambda^{2}} .
$$

Lemma 4. The following integral relation holds true

$$
\int_{0}^{\infty} \frac{x b e i(2 \sqrt{\lambda x})}{x^{2}+\eta^{2}} d x=\frac{\pi}{2} I_{0}(2 \sqrt{\lambda \eta}) .
$$

Proof. Let us consider the following integral

$$
\mathscr{I}(\lambda)=\int_{0}^{\infty} \frac{x b e i(2 \sqrt{\lambda x})}{x^{2}+\eta^{2}} d x
$$

Taking Laplace transform of the above function with respect to $\lambda$, leads to

$$
\mathscr{L}\{\mathscr{I}(\lambda\})=\int_{0}^{\infty} e^{-s \lambda}\left(\int_{0}^{\infty} \frac{x b e i(2 \sqrt{\lambda x})}{x^{2}+\eta^{2}} d x\right) d \lambda
$$

changing the order of integration which is permissible, yields

$$
\mathscr{L}\{\mathscr{I}(\lambda\})=\int_{0}^{\infty} \frac{x}{x^{2}+\eta^{2}}\left(\int_{0}^{\infty} e^{-s \lambda} \operatorname{bei}(2 \sqrt{x \lambda}) d \lambda\right) d x
$$

The value of inner integral is $\frac{1}{s} \sin \left(\frac{x}{s}\right)$, therefore we have

$$
\mathscr{L}\{\mathscr{I}(\lambda\})=\int_{0}^{\infty} \frac{x}{x^{2}+\eta^{2}}\left(\frac{1}{s} \sin \left(\frac{x}{s}\right)\right) d x
$$

The above integral can be evaluated by means of calculus of residues to obtain

$$
\mathscr{L}\{\mathscr{I}(\lambda\})=\frac{\pi}{2} \frac{e^{\frac{\eta}{s}}}{s}
$$


At this point, taking the inverse Laplace transform of the above relation to get

$$
\mathscr{I}(\lambda\}=\frac{\pi}{2} I_{0}(2 \sqrt{\lambda \eta})
$$

Lemma 5. Let us assume that

$$
\mathscr{L}\left\{\left(\frac{t}{a}\right)^{\frac{v}{2}} J_{v}(2 \sqrt{a t}\}=\frac{e^{-\frac{a}{s}}}{s^{v+1}}\right.
$$

then we have the following integral relation

$$
\begin{gathered}
\int_{0}^{\frac{\pi}{2}}\left(\frac{\sqrt{t} \cos \phi}{\sqrt{a}}\right)^{v} J_{v}(2 \sqrt{a t} \cos \phi)\left(\frac{\sqrt{t} \sin \phi}{\sqrt{b}}\right)^{\mu} J_{\mu}(2 \sqrt{b t} \sin \phi) \sin 2 \phi d \phi=. . \\
\left(\frac{t}{a+b}\right)^{\frac{v+\mu+2}{2}} J_{v+\mu+2}(2 \sqrt{(a+b) t}) .
\end{gathered}
$$

Proof. By using convolution theorem for the Laplace transforms, we have the following relation

$$
\mathscr{L}^{-1}\left\{\left(\frac{e^{-\frac{a}{s}}}{s^{v+1}}\right)\left(\frac{e^{-\frac{b}{s}}}{s^{\mu+1}}\right)\right\}=\left(\frac{t}{a}\right)^{\frac{v}{2}} J_{v}(2 \sqrt{a t}) *\left(\frac{t}{b}\right)^{\frac{\mu}{2}} J_{\mu}(2 \sqrt{b t})=\mathscr{L}^{-1}\left\{\left(\frac{e^{-\frac{a+b}{s}}}{s^{v+\mu+2}}\right)\right.
$$

or,

$$
\left(\frac{t}{a}\right)^{\frac{v}{2}} J_{v}(2 \sqrt{a t}) *\left(\frac{t}{b}\right)^{\frac{\mu}{2}} J_{\mu}(2 \sqrt{b t})=\int_{0}^{t}\left(\frac{t-\xi}{a}\right)^{\frac{v}{2}} J_{v}(2 \sqrt{a(t-\xi)})\left(\frac{\xi}{b}\right)^{\frac{\mu}{2}} J_{\mu}(2 \sqrt{b \xi}) d \xi .
$$

Let us introduce a change of variable $t \sin ^{2} \phi=\xi$, we obtain

$$
\begin{gathered}
\int_{0}^{\frac{\pi}{2}}\left(\frac{\sqrt{t} \cos \phi}{\sqrt{a}}\right)^{v} J_{v}(2 \sqrt{a t} \cos \phi)\left(\frac{\sqrt{t} \sin \phi}{\sqrt{b}}\right)^{\mu} J_{\mu}(2 \sqrt{b t} \sin \phi) \sin 2 \phi d \phi=\ldots \\
=\left(\frac{t}{a+b}\right)^{\frac{v+\mu+2}{2}} J_{v+\mu+2}(2 \sqrt{(a+b) t}) .
\end{gathered}
$$

Singular integral equations arise in many problems of mathematical physics. The mathematical formulation of physical phenomenon often involves Cauchy types, or singular integral equations. Singular integral equations have many applications in important fields, like fracture mechanics, elastic contact problems, the theory of porous filtering contain integral and integro - differential equations with singular kernel.

Theorem 2. Let us consider the following system of fractional Volterra integro-differential equations of convolution-type with the Bessel kernel

$$
\begin{aligned}
& { }_{0}^{C} D_{t}^{\alpha} \phi(x)=f_{1}(x)+\lambda \int_{0}^{t}\left(\frac{x-t}{a}\right)^{\frac{v}{2}} I_{v}(2 \sqrt{a t}) \psi(t) d t, \\
& { }_{0}^{C} D_{x}^{\beta} \psi(x)=f_{2}(x)+\eta \int_{0}^{x}\left(\frac{x-t}{b}\right)^{\frac{\mu}{2}} J_{\mu}(2 \sqrt{b t}) \phi(t) d t,
\end{aligned}
$$

where $\phi(0)=\psi(0)=0$ and $0<\alpha, \beta \leq 1$, and $f_{1}(x), f_{2}(x)$ are known functions. Then the above system has the following formal solutions

$$
\begin{gathered}
\phi(t)=\sum_{k=0}^{\infty}(\lambda \eta)^{k} f_{1}(t) *\left\{\left(\frac{t}{k(a+b)}\right)^{\frac{k \sigma+\alpha-1}{2}} J_{k \sigma+\alpha-1}(2 \sqrt{k(a+b) t}\}+\ldots\right. \\
+\sum_{k=0}^{\infty}(\lambda \eta)^{k} f_{2}(t) *\left\{\left(\frac{t}{k(a+b)+a}\right)^{\frac{k \sigma+\tau+\mu-1}{2}} J_{k \sigma+\tau+\mu-1}(2 \sqrt{(k(a+b)+a) t}\}\right.
\end{gathered}
$$

and

$$
\psi(t)=\sum_{k=0}^{\infty}(\lambda \eta)^{k} f_{2}(t) *\left\{\left(\frac{t}{k(a+b)}\right)^{\frac{k \sigma+\beta-1}{2}} J_{k \sigma+\beta-1}(2 \sqrt{k(a+b) t}\}+\ldots\right.
$$




$$
+\sum_{k=0}^{\infty}(\lambda \eta)^{k} f_{1}(t) *\left\{\left(\frac{t}{k(a+b)+b}\right)^{\frac{k \sigma+\tau+v-1}{2}} J_{k \sigma+\tau+\mu-1}(2 \sqrt{(k(a+b)+b) t}\} .\right.
$$

Note. In the above equations, fractional derivitive is in the Caputo sense.

Proof. By applying the Laplace transform on both sides of the above equations term - wise, after simplifying we get the following

$$
\begin{aligned}
& s^{\alpha} \Phi(s)=F_{1}(s)+\lambda \frac{e^{-\frac{a}{s}}}{s^{v+1}} \Psi(s), \\
& s^{\beta} \Psi(s)=F_{2}(s)+\eta \frac{e^{-\frac{b}{s}}}{s^{\mu+1}} \Phi(s) .
\end{aligned}
$$

By solving the above system we get the following

$$
\begin{gathered}
\Phi(s)=\frac{F_{1}(s)+\frac{\lambda e^{-\frac{a}{s}}}{s^{v+\beta+1}} F_{2}(s)}{s^{\alpha}-\frac{\lambda \eta e^{-\frac{a+b}{s}}}{s^{\nu+\beta+\mu+1}}} \\
\Psi(s)=\frac{F_{1}(s)+\frac{\eta e^{-\frac{b}{s}}}{s^{\mu+\alpha+1}} F_{1}(s)}{s^{\beta}-\frac{\lambda \eta e^{-\frac{a+b}{s}}}{s^{v+\alpha+\mu+1}}}
\end{gathered}
$$

by using series expantions, we obtain

$$
\begin{gathered}
\Phi(s)=F_{1}(s) \sum_{k=0}^{\infty} \frac{(\lambda \eta)^{k} e^{-\frac{k(a+b)}{s}}}{s^{k \sigma+\alpha}}+F_{2}(s) \sum_{k=0}^{\infty} \frac{(\lambda \eta)^{k} e^{-\frac{k(a+b)+a}{s}}}{s^{k \sigma+\tau+v}} \\
\Psi(s)=F_{2}(s) \sum_{k=0}^{\infty} \frac{(\lambda \eta)^{k} e^{-\frac{k(a+b)}{s}}}{s^{k \delta+\beta}}+F_{1}(s) \sum_{k=0}^{\infty} \frac{(\lambda \eta)^{k} e^{-\frac{k(a+b)+b}{s}}}{s^{k \delta+\tau+\mu}} \\
\sigma=v+\mu+\beta+2, \delta=v+\mu+\alpha=2, \tau=\alpha+\beta+1 .
\end{gathered}
$$

Now, using the fact that

$$
\mathscr{L}\left\{\left(\frac{t}{a}\right)^{\frac{v}{2}} J_{v}(2 \sqrt{a t}\}=\frac{e^{-\frac{a}{s}}}{s^{v+1}},\right.
$$

by taking the inverse Laplace transform of the above relations term wise, we arrive at

$$
\begin{gathered}
\phi(t)=\sum_{k=0}^{\infty}(\lambda \eta)^{k} f_{1}(t) *\left\{\left(\frac{t}{k(a+b)}\right)^{\frac{k \sigma+\alpha-1}{2}} J_{k \sigma+\alpha-1}(2 \sqrt{k(a+b) t}\}+\ldots\right. \\
+\sum_{k=0}^{\infty}(\lambda \eta)^{k} f_{2}(t) *\left\{\left(\frac{t}{k(a+b)+a}\right)^{\frac{k \sigma+\tau+\mu-1}{2}} J_{k \sigma+\tau+\mu-1}(2 \sqrt{(k(a+b)+a) t}\}\right.
\end{gathered}
$$

and

$$
\begin{gathered}
\psi(t)=\sum_{k=0}^{\infty}(\lambda \eta)^{k} f_{2}(t) *\left\{\left(\frac{t}{k(a+b)}\right)^{\frac{k \sigma+\beta-1}{2}} J_{k \sigma+\beta-1}(2 \sqrt{k(a+b) t}\}+\ldots\right. \\
+\sum_{k=0}^{\infty}(\lambda \eta)^{k} f_{1}(t) *\left\{\left(\frac{t}{k(a+b)+b}\right)^{\frac{k \sigma+\tau+v-1}{2}} J_{k \sigma+\tau+\mu-1}(2 \sqrt{(k(a+b)+b) t}\} .\right.
\end{gathered}
$$




\section{Solution to impulsive KdV via Joint Laplace - Fourier transforms}

In this section, the author implemented the joint Laplace Fourier transform to construct exact solution for a variety of the non - homogeneous $\mathrm{KdV}$ equation. The $\mathrm{KdV}$ equations are attracting many researchers around the world and a great deal of work has already been done in some of these equation.

$$
\begin{aligned}
u_{t}+\alpha u+k u_{x}+\lambda u_{x x x} & =\beta \delta(t) A i(x), \\
u(x, 0) & =\phi(x) .
\end{aligned}
$$

Then the above equation has the following formal solution. Note that $A i($.$) stands for the Airy function.$

Solution 2. By taking the joint Laplace - Fourier transform of the above equation and using boundary condition, we get the following transformed equation

$$
\hat{\bar{U}}(w, s)=\frac{\Phi(w)}{s+\left(i k w-i \gamma w^{3}+\alpha\right)}+\frac{\beta G(w)}{s+\left(i k w-i \gamma w^{3}+\alpha\right)} .
$$

For the sake of simplicity, let us assume that $\tau=i k w-i \gamma w^{3}+\alpha$, and using inverse Laplace transform of transformed equation to obtain

$$
\hat{U}(w, t)=\mathscr{L}^{-1}\left\{\frac{\Phi(w)+\beta G(w)}{s+\tau} ; s->t\right\}
$$

or,

$$
\hat{U}(w, t)=(\Phi(w)+\beta G(w)) e^{-t \tau}
$$

At this point, inverting Fourier transform to get

$$
u(x, t)=\frac{1}{\sqrt{2 \pi}} \int_{-\infty}^{+\infty} \exp (-i x w)(\Phi(w)+\beta G(w)) e^{-t \tau} d w
$$

By setting $\tau=i k w-i \gamma w^{3}+\alpha$, and after simplifying we get

$$
u(x, t)=\frac{e^{-\alpha t}}{\sqrt{2 \pi}} \int_{-\infty}^{+\infty} \exp (-i(k t-x) w)(\Phi(w)+\beta G(w)) e^{i t \gamma w^{3}} d w
$$

or, equivalently

$$
u(x, t)=\frac{e^{-\alpha t}}{\sqrt{2 \pi}} \int_{-\infty}^{+\infty}(\phi(\eta)+\beta A i(\eta)) A i\left(\frac{\eta-k t+x}{\sqrt[3]{3 \gamma \eta}}\right) d \eta
$$

Note: For special case

$$
\beta=\alpha=0, \gamma=\frac{1}{3}
$$

We get the simple standard linearized $\mathrm{KdV}$, with the following solution

$$
u(x, t)=\frac{1}{\sqrt{2 \pi}} \int_{-\infty}^{+\infty} \phi(\eta) A i\left(\frac{\eta-k t+x}{\sqrt[3]{\eta}}\right) d \eta
$$




\section{Main Results}

Problem 1. Coupled Non-Homogenous Sub- Ballistic Fractional PDE Solve the following coupled non - homogeneous partial fractional differential equations with constant coefficients. The special case of sub- ballistic fractional PDE considered by V.V.Uchaikin [21].

$$
\begin{gathered}
D_{t}^{c, \alpha} u+\frac{\partial u}{\partial x}-k v=\phi(x),(3.1) \quad D_{t}^{c, \alpha} v+\frac{\partial v}{\partial x}+k u=\psi(x) . \\
u(x, 0)=0, \quad v(0, t)=\beta_{1} \quad v(x, 0)=0, \quad v(0, t)=\beta_{2} .
\end{gathered}
$$

Solution 3. We define the Laplace - transform of $u(x, t)$ by

$$
U(x, s)=\int_{0}^{\infty} u(x, t) e^{-s t} d t
$$

Let us define $w(x, t)=u(x, t)+i v(x, t), w(0, t)=u(0, t)+i v(0, t)=\beta, \phi(x)+i \psi(x)=h(x)$ and $w(x, 0)=u(x, 0)+$ $i v(x, 0)=0$, then the above system is equivalent to the following

$$
\begin{aligned}
& D_{t}^{c, \alpha} w+\frac{\partial w}{\partial x}+i k w=h(x), \\
& w(x, 0)=0, w(0, t)=\beta .
\end{aligned}
$$

Application of the Laplace transform leads to the solution of the transformed problem in the following form

$$
\frac{\partial W}{\partial x}+\left(s^{\alpha}+i k\right) W=\frac{h(x)}{s}
$$

The above first order differential equation has the following solution

$$
W(x, s)=\frac{\beta}{s} e^{-\left(s^{\alpha}+i k\right) x}+\frac{e^{-\left(s^{\alpha}+i k\right) x}}{s} \int_{0}^{x} h(\eta) e^{\left(s^{\alpha}+i k\right) \eta} d \eta,
$$

at this point, we set $\alpha=0.5$ ( semi derivative case) in the above relation, to obtain

$$
W(x, s)=\frac{\beta}{s} e^{-(\sqrt{s}+i k) x}+\frac{e^{-(\sqrt{s}+i k) x}}{s} \int_{0}^{x} h(\eta) e^{(\sqrt{s}+i k) \eta} d \eta
$$

taking the inverse Laplace transform to obtain

$$
u(x, t)+i v(x, t)=\beta e^{-i k x} \operatorname{Erfc}\left(\frac{x}{2 \sqrt{t}}\right) \int_{0}^{x} e^{-i k(x-\xi} h(\xi) \operatorname{Erfc}\left(\frac{x-t}{2 \sqrt{\xi}}\right) d \xi
$$

now, taking real and imaginary part of the above relationship to obtain

$$
u(x, t)=\left(\beta_{1} \cos k x+\beta_{2} \sin k x\right) \operatorname{Erfc}\left(\frac{x}{2 \sqrt{t}}\right)+\ldots+\int_{0}^{x}(\phi(\xi) \cos k(x-\xi)+\psi(\xi) \sin k(x-\xi)) \operatorname{Erfc}\left(\frac{x-t}{2 \sqrt{\xi}}\right) d \xi
$$

and

$$
v(x, t)=\left(\beta_{2} \cos k x-\beta_{1} \sin k x\right) \operatorname{Erfc}\left(\frac{x}{2 \sqrt{t}}\right)+\ldots+\int_{0}^{\infty}(\psi(\xi) \cos k(x-\xi)-\phi(\xi) \sin k(x-\xi)) \operatorname{Erfc}\left(\frac{x-t}{2 \sqrt{\xi}}\right) .
$$


For special case $\phi(x)=\delta(x-\lambda), \psi(x)=\delta(x-\mu)$, we get finally the following results

$$
\left.\left.u(x, t)=\left(\beta_{1} \cos k x+\beta_{2} \sin k x\right) \operatorname{Erfc}\left(\frac{x}{2 \sqrt{t}}\right)+\ldots+\cos k(x-\lambda)\right) \operatorname{Erfc}\left(\frac{x-t}{2 \sqrt{\lambda}}\right) \sin k(x-\mu)\right) \operatorname{Erfc}\left(\frac{x-t}{2 \sqrt{\mu}}\right),
$$

and

$$
v(x, t)=\left(\beta_{2} \cos k x-\beta_{1} \sin k x\right) \operatorname{Erfc}\left(\frac{x}{2 \sqrt{t}}\right)+\ldots+\left(\cos k(x-\mu) \operatorname{Erfc}\left(\frac{x-t}{2 \sqrt{\mu}}-\sin k(x-\lambda)\right) \operatorname{Erfc}\left(\frac{x-t}{2 \sqrt{\lambda}}\right) .\right.
$$

\section{Conclusions}

The paper is devoted to study and application of the Laplace transform for solving certain system of time fractional partial differential equations and evaluation of certain integrals of Besselâ's functions. The author considered a generalization of the problem of sub- ballistic fractional PDE studied by V.V.Uchaikin. The transform method provides powerful method for analyzing linear systems. The main purpose of this work is to develop a method for finding analytic solution of the system of integro-differential equations and time fractional PDEs.

\section{Ackknowledgments}

The author would like to thank the refree/s and editor/s for careful and thoughtful readings of the manuscript which helped to improve the presentation of the results.

\section{Competing interests}

The authors declare that they have no competing interests.

\section{Authors' contributions}

All authors have contributed to all parts of the article. All authors read and approved the final manuscript.

\section{References}

[1] A. Aghili, H. Zeinali. Advances in Laplace type integral transforms with applications. Indian Journal of Science and Technology, Vol 7(6), 877-890, June 2014.

[2] A. Aghili, H. Zeinali. Solution to time fractional wave equation in the presence of friction via integral transform. Communications on applied nonlinear analysis, Vol.21, No.2, pp.67-88, 2014.

[3] A. Aghili; M. R. Masomi. Integral transform method for solving different F.S.I.Es and P.F.D.Es. Konuralp Journal of Mathematics, Volume 2, No. 1 pp. 45-62, 2014.

[4] T. M. Atanackovic , B. Stankovic. Dynamics of a visco -elastic rod of Fractional derivative type, Z. Angew. Math. Mech., 82(6), (2002) 377-386.

[5] T. M. Atanackovic , B. Stankovic. On a system of differential equations with fractional derivatives arising in rod theory. Journal of Physics A: Mathematical and General, 37, No 4, 1241-1250 (2004).

[6] D. G. Duffy. Transform methods for solving partial differential equations. Chapman and Hall/CRC, 2004.

[7] R. S. Dahiya. M. Vinayagamoorthy.Laplace transfom pairs of $n$-dimensions and heat conduction problem. Math. Comput. Modelling vol. 13.No. 10 , pp,35-50 
[8] V. A. Ditkin. A. P.Prudnikov. Operational calculus In two variables and its application ,Pergamon Press, New York,1962.

[9] V. W. Ekman. On the influence of the earth's rotation on ocean currents. Ark.Math.Astr.Fys.2.No 1905.

[10] A. A. Kilbass ,J. J. Trujillo. Differential equation of fractional order: methods, results and problems. II, Appl. Anal, 81(2), (2002) 435-493.

[11] Y. Luchko, H. M.Srivastava.The exact solution of certain differential equations of fractional Order by using operational calculus.Comput.Math.Appl.29 (1995)73-85

[12] S. Miller, B. Ross. An introduction to fractional differential equations, Wiley, NewYork.

[13] K. B. Oldham, J. Spanier.The Fractional calculus,Academic Press, NewYork, 1974.

[14] K. B. Oldham, J. Spanier, Fractional calculus and its applications, Bull.Inst.Politech.. Sect. I, 24 (28)(3-4), (1978) 29-34.

[15] I. Podlubny, The Laplace transform method for linear differential equations of fractional order, Slovak Academy of sciences. Slovak Republic, 1994.

[16] I. Podlubny.Fractional differential equations, Academic Press, San Diego, CA, 1999.

[17] G. E. Roberts, H. Kaufman. Table of Laplace transforms, Philadelphia; W.B.Saunders Co. 1966.

[18] G. Samko , A. Kilbas. O.Marchiev. Fractional Integrals and derivatives theory and applications. Gordon and Breach,Amesterdam,1993.

[19] W. Schneider, W. Wyss. Fractional diffusion and wave equations. J. Math. Phys.30(1989)134-144.

[20] B. A. Stankovic. system of partial differential equations with fractional derivatives,Math. Vesnik, 3-4(54), (2002) 187-194.

[21] V. V. Uchaikin. Fractional derivatives for physicists and engineers.vol.1.Springer 2012.

[22] W. Wyss. The fractional diffusion equation, J. Math. Phys., 27(11), (1986) 2782-2785. 